\title{
BMJ Open Geriatric CO-mAnagement for Cardiology patients in the Hospital (G-COACH): study protocol of a prospective before-after effectiveness- implementation study
}

Mieke Deschodt, ${ }^{1,2}$ Bastiaan Van Grootven, ${ }^{3,4}$ Anthony Jeuris, ${ }^{4}$ Els Devriendt, ${ }^{5}$ Bernadette Dierckx de Casterlé, ${ }^{4}$ Christophe Dubois, ${ }^{6}$ Katleen Fagard, ${ }^{5}$ Marie-Christine Herregods, ${ }^{6}$ Miek Hornikx, ${ }^{6}$ Bart Meuris, ${ }^{6}$ Steffen Rex, ${ }^{7}$ Jos Tournoy, ${ }^{1,5}$ Koen Milisen, ${ }^{5}$ Johan Flamaing ${ }^{1,5}$

To cite: Deschodt M, Van Grootven B, Jeuris A, et al. Geriatric $\mathrm{CO}$-mAnagement for Cardiology patients in the Hospital (G-COACH): study protocol of a prospective before-after effectivenessimplementation study. BMJ Open 2018;8:e023593. doi:10.1136/

bmjopen-2018-023593

- Prepublication history for this paper is available online To view these files, please visit the journal online (http://dx.doi. org/10.1136/bmjopen-2018023593).

$M D$ and $B V G$ contributed equally.

Received 18 April 2018

Revised 3 September 2018

Accepted 6 September 2018

Check for updates

(C) Author(s) (or their employer(s)) 2018. Re-use permitted under CC BY-NC. No commercial re-use. See rights and permissions. Published by BMJ.

For numbered affiliations see end of article.

Correspondence to Dr Mieke Deschodt; mieke.deschodt@kuleuven.be

\section{ABSTRACT}

Introduction Although the majority of older patients admitted to a cardiology unit present with at least one geriatric syndrome, guidelines on managing heart disease often do not consider the complex needs of frail older patients. Geriatric co-management has demonstrated potential to improve functional status, and reduce complications and length of stay, but evidence on the effectiveness in cardiology patients is lacking. This study aims to determine if geriatric co-management is superior to usual care in preventing functional decline, complications, mortality, readmission rates, reducing length of stay and improving quality of life in older patients admitted for acute heart disease or for transcatheter aortic valve implantation, and to identify determinants of success for geriatric co-management in this population.

Methods and analysis This prospective quasiexperimental before-and-after study will be performed on two cardiology units of the University Hospitals Leuven in Belgium in patients aged $\geq 75$ years. In the precohort $(n=227)$, usual care will be documented. A multitude of implementation strategies will be applied to allow for successful implementation of the model. Patients in the after cohort $(\mathrm{n}=227)$ will undergo a comprehensive geriatric assessment within 24 hours of admission to stratify them into one of three groups based on their baseline risk for developing functional decline: low-risk patients receive proactive consultation, high-risk patients will be co-managed by the geriatric nurse to prevent complications and patients with acute geriatric problems will receive an additional medication review and comanagement by the geriatrician.

Ethics and dissemination The study protocol was approved by the Medical Ethics Committee UZ Leuven/ KU Leuven (S58296). Written voluntary (proxy-)informed consent will be obtained from all participants at the start of the study. Dissemination of results will be through articles in scientific and professional journals both in English and Dutch and by conference presentations.

Trial registration number NCT02890927.

\section{Strengths and limitations of this study}

- A geriatric co-management intervention theory was developed to increase the a priori probability for a clinically meaningful effect.

- Stakeholder involvement in the development, feasibility and evaluation phase facilitates the implementation of a care programme that fits the local context and is deemed acceptable and feasible by all stakeholders.

- Exploration of components that contributed to the successful implementation using a mixed-methods approach will inform scaling up and out of the care model.

- Because of the inability to randomise individual patients in this single-centre study, there is a risk of residual confounding.

\section{INTRODUCTION}

Longevity is the result of improved population health, but at the same time leads to an absolute increase of people suffering from multiple chronic health problems and disability. ${ }^{1}$ The complex care for these patients is hampered by the high prevalence of frailty, cognitive impairment and functional dependency, which has been associated with functional decline, increased mortality, hospital readmission and need for new social support. ${ }^{2-4}$ Concurringly, the majority of healthcare staff is not adequately trained to manage the complex geriatric needs of these older patients. ${ }^{5}$ Inappropriate medication use, delirium, cognitive impairment and depression are often not recognised in older patients, emphasising the need for better geriatric care. ${ }^{6-10}$ 
Cardiovascular disease is the leading cause of death and hospitalisation in the Western world. ${ }^{11}$ Notably, the majority of older patients admitted to a cardiology unit present with at least one geriatric syndrome. ${ }^{2}$ Current evidence-based guidelines on the management of heart disease often do not consider the complex needs of frail older patients, and may even incur harm. ${ }^{12}$ This has prompted researchers and clinicians to advocate for a closer collaboration between cardiology and geriatric medicine as the "management of cardiac issues is fundamentally linked to the frailties and multimorbidities associated with advanced age'. ${ }^{12} 13$

Comprehensive geriatric assessment (CGA) has previously been identified as the gold standard for managing geriatric patients, but has not yet been evaluated in older cardiology patients. ${ }^{14}$ CGA refers to a 'multidimensional, interdisciplinary diagnostic process to determine the medical, psychological and functional capabilities of an older person with frailty, followed by implementation of a coordinated and integrated plan for treatment and follow-up' ${ }^{15}$ A model of care that embeds the principles of CGA is the geriatric consultation team model. Geriatric consultation teams are multidisciplinary mobile teams that assess older patients admitted to non-geriatric units and recommend a plan of treatment. However, a meta-analysis detected no significant effect on functional status, length of stay and readmission and only found a moderate beneficial effect on mortality at 6 and 8 months after hospitalisation. ${ }^{16}$ Subsequently, geriatric co-management programmes have emerged as a new model of CGA-based care for non-geriatric units.

Geriatric co-management is defined as a shared responsibility and decision making between at least a primary treating physician (eg, cardiologist) and a geriatrician or geriatric team who provides complementary medical care in the prevention and management of geriatric problems. ${ }^{17}$ A recent meta-analysis observed a better functional status, a decrease in complications and a reduced length of stay in favour of co-managed patients. ${ }^{18}$ These results confirm the potential value of geriatric co-management, and indicate a need to further evaluate the concept due to the low quality of evidence. Furthermore, only four studies with inconsistent results assessed functional status as outcome and the majority of studies were performed in orthopaedic patients. ${ }^{18}$ There is currently no evidence on the effectiveness of geriatric co-management in older cardiology patients.

This protocol is part of the CO-mAnagement for Cardiology patients in the Hospital (G-COACH) project, which aims to develop and evaluate an in-hospital cardiogeriatric co-management model using a mixed-methods multiphase methodology. The aim of this paper is to present a detailed overview of the methodology of the G-COACH feasibility and effectiveness study, based on the Standard Protocol Items: Recommendations for Interventional Trials statement. ${ }^{19}$

\section{METHODOLOGY}

\section{Methodological framework}

The G-COACH project is based on the Medical Research Council (MRC) framework for the development and evaluation of complex interventions (figure 1) ${ }^{20}$ As part of the development phase of the MRC framework and in preparation of the feasibility and evaluation studies, we first developed an intervention theory for geriatric co-management that details how the G-COACH intervention will affect the desired change in outcomes. This theory was developed by integrating evidence from (1) a systematic review and meta-analysis on the effectiveness of geriatric co-management programmes, ${ }^{18}$ (2) an international Delphi study that aimed to find consensus on appropriate and feasible structure, process and outcome indicators for the evaluation of in-hospital geriatric co-management programmes ${ }^{21}$ and (3) an exploratory prospective cohort study in hospitalised patients with cardiac conditions to determine the incidence of in-hospital functional decline, the associated risk factors and the link with care processes. ${ }^{22}$ Additionally, we developed a clinical prediction model that identifies patients who are at risk for developing functional decline during hospitalisation. This risk prediction model was built based on data from the precohort of this intervention study, and will be used to identify patients in need for geriatric co-management, that is, patients with an increased risk for functional decline (submitted manuscript). To the best of our knowledge, no such model is available for older patients admitted to an acute cardiac care unit. The model will be validated in a cohort of 189 patients aged 75 years or older who are admitted to an acute cardiac care unit. Non-parametric bootstrapping will be used for internal validation.

The G-COACH feasibility and effectiveness study described in this paper concerns phase II and phase III of the MRC framework. However, to substantially increase the likelihood that the evaluated geriatric co-management programme moves from trial to real world, we use a hybrid 1 effectiveness-implementation design. ${ }^{23}$ This means that in parallel with evaluating the effectiveness of the geriatric co-management model, we will gather information to inform future implementation strategies for scaling up and scaling out the geriatric co-management model. Hence, while trying to get an in-depth understanding of which intervention components are effective and which are not, we aim to provide a comprehensive overview of barriers and facilitators for large-scale implementation of the care model following its evaluation. The latter will be done by considering contextual factors that may influence the success of the implementation and the variation in outcomes from the very beginning of the project and by actively involving stakeholders in each project phase. ${ }^{23}$

\section{Study aims}

The overall aim of the feasibility study is to (1) assess reach, fidelity and dose of the intervention; (2) investigate the 


\section{MRC phase I: Development}

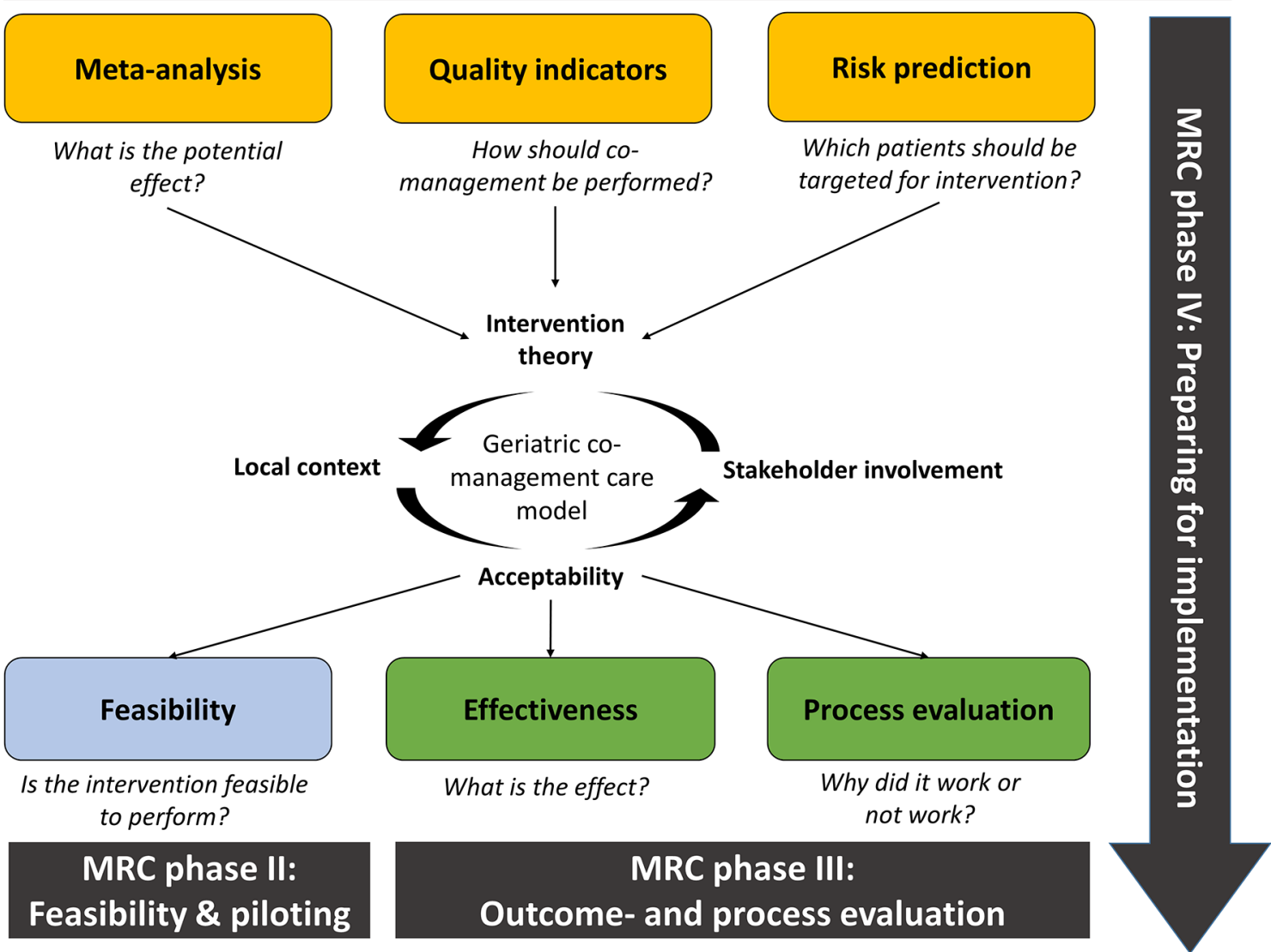

Figure 1 Overview of the CO-mAnagement for Cardiology patients in the Hospital project. MRC, Medical Research Council.

perceived acceptability of the intervention by healthcare professionals and patients participating in the intervention and (3) determine facilitators and barriers for the implementation of the intervention.

The overall aim of the effectiveness study is twofold. The outcome evaluation will determine if geriatric co-management is superior in preventing in-hospital functional decline (primary outcome) and complications, reducing length of stay, decreasing mortality and readmission rates and improving quality of life in older patients admitted for acute heart disease or for transcatheter aortic valve implantation (TAVI) compared with usual care. The process evaluation will determine the quality of the implementation by investigating how well the fidelity and dose is maintained during the study period and how the geriatric co-management programme is adapted over time due to interaction with the local context. ${ }^{24}$

\section{Design and setting}

This single-centre, prospective, quasi-experimental before-and-after study will be performed on two cardiology units of the University Hospitals Leuven in Belgium. The University Hospitals Leuven is one of the seven university and tertiary hospitals in Belgium, and has 1995 beds. The two general cardiology units consist of 44 hospitalisation beds. Between recruiting patients in the before and after cohorts, the geriatric co-management intervention will be implemented and piloted to assess its feasibility.

\section{Study population}

Dutch-speaking patients aged 75 years or over are included if they are admitted through the emergency department or cardiology outpatient services for non-surgical treatment of acute heart disease or TAVI, have an expected length of stay of $\geq 3$ days and give (proxy) informed consent. Patients are excluded if they are admitted from another hospital or hospital unit (no baseline data for functional status), if they stay in the intensive care unit for 3 days or longer (healthcare professionals on these wards are not involved in the development of the geriatric co-management intervention and/or impossibility to execute core components of the intervention, eg, mobility protocols) or if they receive palliative treatment on hospital admission.

\section{Usual care}

The control group receives usual care on the cardiology units. Team members include a cardiology or internal medicine resident supervised by a consultant cardiologist, ward nurses, a physiotherapist, a social worker and a dietician, who meet weekly at a multidisciplinary team meeting. A geriatric support team, consisting of seven 


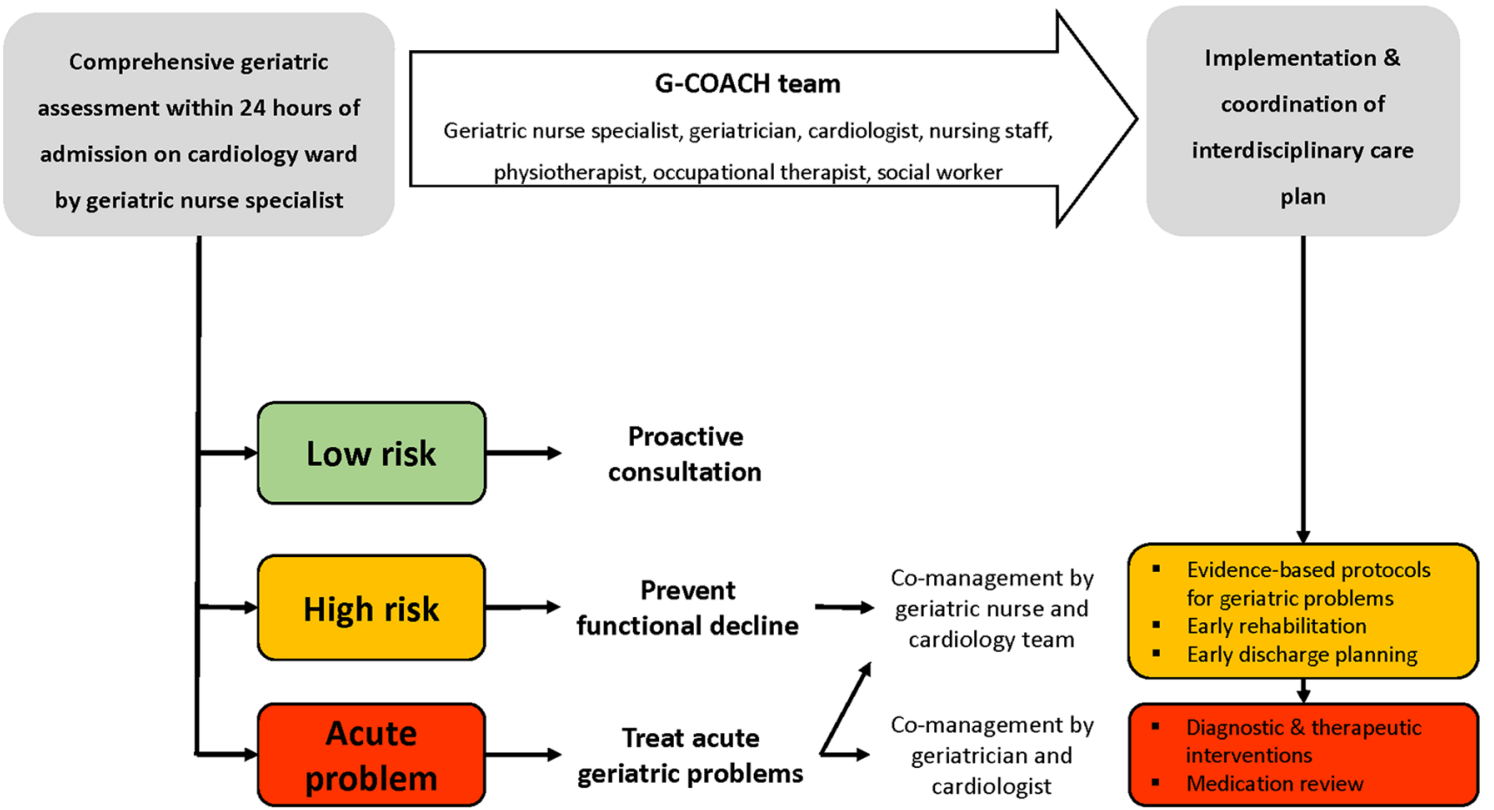

Figure 2 Overview of the CO-mAnagement for Cardiology patients in the Hospital (G-COACH) intervention.

geriatric nurses (3.8 full-time equivalents (FTE) including one master-trained nurse), a master-trained head nurse (1 FTE), four occupational therapists (2 FTE) and two geriatricians (0.2 FTE), is available for consultation services on request of all non-geriatric wards in the study hospital, including the cardiology wards. If consulted, the geriatric support team performs a CGA and gives written and oral recommendations about detected geriatric problems. ${ }^{25}$

\section{Geriatric co-management intervention}

Every weekday a geriatric nurse is responsible for the geriatric co-management patients and conducts a CGA within 24 hours of admission in eligible patients newly admitted to the cardiology unit (figure 2). Subsequently, patients are stratified into one of three groups based on their baseline risk for developing functional decline. This risk prediction considers cognitive impairment (Mini-Cog score), mobility impairment (use of ambulatory aid), nutritional risk status (Mini Nutritional Assessment (MNA) score), depressive symptoms (Geriatric Depression Scale score) and the presence of physical restraint use or an indwelling urinary catheter (data not yet published).

Low-risk patients are patients who are at low risk for developing functional decline during hospitalisation. The geriatric nurse provides a proactive consultation without systematic follow-up.

High-risk patients are at risk for developing functional decline and other geriatric complications during hospitalisation. The geriatric nurse will work collaboratively with the cardiology team to prevent complications. Interventions include care coordination and bedside education by the geriatric nurse, early rehabilitation by a physical therapist, early discharge planning by a social worker and availability of evidence-based protocols for the prevention and/or management of functional decline, falls, delirium, cognitive impairment, agitation, malnutrition, urinary incontinence, urinary retention, urinary tract infection, obstipation, pressure sores and pain. All intervention components selected from the protocols are tailored to the specific needs of an individual patient as detected with the CGA on admission. The geriatric nurse provides daily follow-up and coordinates the implementation of the protocols.

Patients with acute geriatric problems have developed one of the following geriatric syndromes: agitation, delirium, urinary retention, urinary incontinence or malnutrition (MNA score $<8 / 14$ ) and are subsequently considered to be at high risk of developing functional decline. These patients receive the same care as the high-risk patients. Additionally, the geriatrician will perform a medication review based on clinical expertise and will co-manage the delirium, urinary retention, urinary incontinence and/or malnourishment with the cardiologist.

\section{Implementation strategies}

Changing the organisation and daily activities of a geriatric support team that has been working as a consultation team since 2005 is challenging. Both the geriatric support team and the healthcare professionals of the cardiology units need to take up a new role with new responsibilities and competencies. Since the aim is to change behaviour in both the geriatric support team and the cardiology teams, we use the Intervention Mapping taxonomy of behaviour change methods to ensure that our applied implementation strategies were targeting determinants that predict behaviour and were able to actually change that determinant. ${ }^{26}$ Table 1 gives a detailed overview of the targeted determinants and practical strategies to change behaviour in the geriatric support and cardiology team. 
Table 1 Implementation strategies and related behaviour change methods

\begin{tabular}{lll}
\hline Process & Determinant and aim & Strategy \\
Orientation & $\begin{array}{l}\text { Knowledge: stakeholders are aware of the co- } \\
\text { management programme }\end{array}$ & $\begin{array}{l}\text { Listing all relevant stakeholders in the organisation } \\
\text { Stakeholder meetings in initiation phase to propose programme } \\
\text { with head of departments of geriatrics, cardiology, nursing, } \\
\text { physiotherapy, nutritional therapy, social work and with head } \\
\text { nurses of cardiology and geriatric support team, care programme } \\
\text { managers and informations and communications technology }\end{array}$ \\
& $\begin{array}{l}\text { Use of G-COACH acronym in all communication } \\
\end{array}$
\end{tabular}

Taxonomy of behaviour change ${ }^{26}$

Attitude: stakeholders are interested and seek involvement in the co-management programme

Insight

Knowledge: stakeholders understand the goals, concepts and intervention components of the co-management programme

Knowledge: stakeholders understand the geriatric needs of patients admitted to their unit and know the prevalence of geriatric syndromes on hospital admission and the incidence of geriatric complications during hospitalisation

Acceptance
$\begin{aligned} & \text { Positive attitude: healthcare professionals } \\ & \text { are motivated to work with each other and } \\ & \text { collaborate as one interdisciplinary team } \\ & \text { Self-confidence: stakeholders feel confident that } \\ & \text { participating in the co-management programme } \\ & \text { is feasible and that any problems arising will be } \\ & \text { solved }\end{aligned}$

Attitude: stakeholders are convinced that the comanagement programme is useful and effective to improve care outcomes for geriatric patients on their units

Attitude: stakeholders have decided to change their standard of care and try out the geriatric co-management programme

Systems change Skills and organisation of new care structures and processes: stakeholders can try the comanagement programme on a small scale and gain experience and skills necessary for the programme

Skills, habits: stakeholders have integrated the co-management programme in their daily care and routines

Qualified staff, self-confidence: stakeholders are adequately staffed and skilled to try out the comanagement programme
Participation

Consciousness raising

Discussion

Participation

Systems change

Chunking

Repeated exposure

Inclusion of stakeholders in consensus-development meetings for Motivational interviewing developing programme, focusing on definition, scope and goals Participation of programme, intervention components and expected benefits

\section{Educational presentations focusing on describing the care}

Active learning

processes and outcomes of the current standard of care and new Advance organisers intervention components that are expected to improve processes Consciousness raising and outcomes. Presentation included case discussion of geriatric Discussion needs and how the programme is expected to improve outcomes Persuasive communication

Inclusion of stakeholders in consensus-development meetings for Participation developing intervention protocols

Intervention manual is available online and in hardcopy to stakeholders

Publication of poster on participating units detailing the programme components and interventions

Situational analysis to document geriatric care needs and the current standard of care by project team

Fact sheets are disseminated and short educational sessions are Consciousness raising repeated in the feasibility and evaluation phase with the purpose Providing cues of disseminating knowledge about geriatric needs to stakeholders Repeated exposure based on the situational analysis

Adaptations to the electronic patient file: risk stratification level and type of follow-up visible for all eligible patients

Facilitation

Providing cues

Technical assistance
Contracting: an expert in group dynamics and leadership organises two sessions between stakeholders
Elaboration

Nudging

Shifting perspective

Inclusion of stakeholders in consensus-development meetings for Nudging developing programme, focusing on definition, scope and goals Participation of programme, intervention components and expected benefits Systems change

The intervention is tailored to match the local context by engaging Elaboration stakeholders to ensure feasibility of the programme

Systems change Tailoring

Participation

Programme support by head of department and head nurses

Fact sheets and short educational sessions are repeated in the feasibility and evaluation phase with focus on impact and positive feedback on achieved goals

Active learning

Advance organisers

Consciousness raising

Repeated exposure

Official start of programme announced by head of departmen

Early commitmen

Persuasive communication

Phased implementation with evaluation of feasibility allowing the programme to adjust if necessary

Active learning

Direct experience
Feedback

Guided practice

Individualisation

Tailoring

Audit and feedback on implementation based on feasibility study Discussion

Feedback

Participatory problem solving

Working group: audit and feedback with key stakeholders from Feedback every discipline to discuss the adaptations that are needed to the Participation programme based on audit and future needs

Participatory problem solving Tailoring

Coaching of geriatric nurses and geriatricians responsible for implementing the programme 


\begin{tabular}{|c|c|c|c|}
\hline Process & Determinant and aim & Strategy & Taxonomy of behaviour change ${ }^{26}$ \\
\hline \multirow[t]{2}{*}{ Maintenance } & $\begin{array}{l}\text { Skills, habits: stakeholders have integrated the } \\
\text { co-management programme in their daily care } \\
\text { and routines }\end{array}$ & $\begin{array}{l}\text { Working group: audit and feedback with key stakeholders from } \\
\text { every discipline to discuss the adaptations that are needed to the } \\
\text { programme based on audit and future needs }\end{array}$ & $\begin{array}{l}\text { Feedback } \\
\text { Participation } \\
\text { Participatory problem solving } \\
\text { Tailoring }\end{array}$ \\
\hline & $\begin{array}{l}\text { Leadership, financial resources, opinion of } \\
\text { leaders and key figures: University Hospitals } \\
\text { Leuven has formally recognised ownership of the } \\
\text { co-management programme }\end{array}$ & $\begin{array}{l}\text { Dissemination of programme results to UZ Leuven staff and } \\
\text { management }\end{array}$ & $\begin{array}{l}\text { Agenda setting } \\
\text { Feedback }\end{array}$ \\
\hline
\end{tabular}

G-COACH, CO-mAnagement for Cardiology patients in the Hospital.

The study coordinator (BVG) and research assistant (AJ) take up the role of external facilitators to allow for successful implementation of the G-COACH intervention. One month before the pilot implementation, they organised information sessions for all stakeholders: nurses, physicians, physiotherapists, occupational therapists, social workers, nutritional therapists and management from both the cardiology and geriatric department. Participants were informed on the current standard of care and the prevalence of geriatric problems. A sense of urgency of why change is needed was created. They were further informed on what will change, how it will change and what the intended benefits will be. Instructional materials, such as an electronic project manual including all intervention protocols, intervention pocket cards and posters, were distributed and training sessions were organised for the geriatric support team to explain and practice the intervention protocols. Finally, a meeting was organised with the external facilitators and geriatric support team to discuss how the team perceives the G-COACH intervention, their specific role and to determine their needs for support towards the external facilitators. This meeting was led by a highly experienced external moderator of the Department of Leadership Development of the University Hospitals Leuven.

At the start of the implementation, an email was sent by the medical head of the departments detailing both the study and instructional materials. The head nurses of the participating units supervised the start of the intervention. A working group was formed consisting of the head nurses of the cardiology units and the geriatric support team, two champion nurses of the cardiology ward, a geriatric expert nurse, cardiologist, geriatrician, physiotherapist, social worker and study coordinator. The purpose of this group that meets monthly, is to discuss the implementation of the intervention, for example, are all intervention components implemented? What are the reasons for non-implementation? What are barriers for implementation? and Are adaptations to the intervention needed? Based on a consensus decision, the working group will propose changes to the intervention or formulate additional implementation strategies.

During the implementation phase, process data will be systematically collected from the electronic patient record and summarised by the study coordinator and research assistant to inform the working group. The study coordinator will organise short informational sessions throughout the study period to inform all stakeholders on the progression and success of the intervention. Weekly updates about the project are sent by mail to the geriatric support team and regular individual feedback sessions with the members of the geriatric support team are organised to emphasise which parts of the implementation of the intervention went well or were challenging.

\section{Patient and public involvement}

Patients and public were not involved in the development of the research questions and outcome measures, the design, recruitment of conduct of the study. Feedback of patients regarding the acceptability of the intervention is actively explored in the feasibility phase of the study using structured patient interviews.

\section{Feasibility evaluation}

The feasibility of the intervention will be assessed in a single intervention group before proceeding to the inclusion of patients in the after cohort. The reach, fidelity (table 2) and dose (table 3) will be evaluated by trained researchers using a multimethods approach.

The reach determines the number of eligible patients that were recruited in the intervention. Successful recruitment is defined as (1) having received CGA and (2) being stratified into a risk group. The number of patients recruited in the intervention will be compared against the number of eligible patients using the electronic patient record. The fidelity determines how well the intervention is implemented as defined by the protocol and considers both the implementation of specific intervention components, and the correct timing of the implementation. The dose determines how much of the intervention is implemented as defined by the protocol and considers both the duration and frequency of specific intervention components. The fidelity and dose will be observed on a daily basis using patient interviews and the electronic patient record.

The experiences of participating healthcare professionals will be captured using focus group discussions or individual interviews. A total of four to five focus groups, including physicians, nurses from the cardiology department and the geriatric support team, physical and occupational therapists and social workers, will be organised. Healthcare professionals not able to participate in the 
Table 2 Fidelity indicators

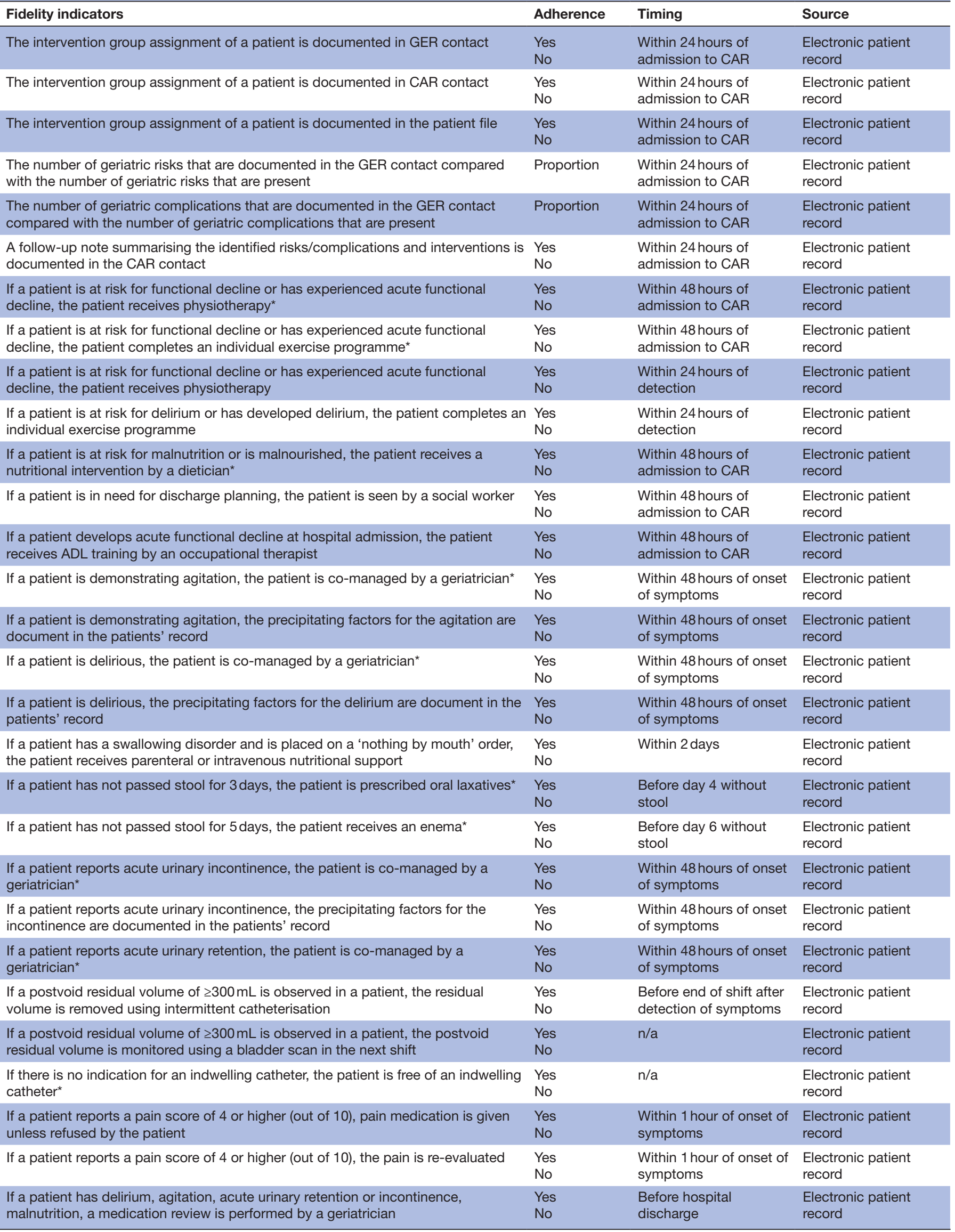

Continued 
Table 2 Continued

\begin{tabular}{|c|c|c|c|}
\hline Fidelity indicators & Adherence & Timing & Source \\
\hline $\begin{array}{l}\text { If a patient has a Mini-Cog score }<3 \text { on hospital admission, a Mini-Mental Status } \\
\text { Examination is performed by an occupational therapist }\end{array}$ & $\begin{array}{l}\text { Yes } \\
\text { No }\end{array}$ & $\begin{array}{l}\text { Before hospital } \\
\text { discharge }\end{array}$ & $\begin{array}{l}\text { Electronic patient } \\
\text { record }\end{array}$ \\
\hline $\begin{array}{l}\text { If a patient is at risk for functional decline, the patient is co-managed by a geriatric } \\
\text { nurse* }\end{array}$ & $\begin{array}{l}\text { Yes } \\
\text { No }\end{array}$ & $\begin{array}{l}\text { Within } 48 \text { hours of } \\
\text { admission to CAR }\end{array}$ & $\begin{array}{l}\text { Electronic patient } \\
\text { record }\end{array}$ \\
\hline $\begin{array}{l}\text { If a patient has delirium, agitation or acute urinary retention or incontinence, the } \\
\text { patient is co-managed by a geriatric nurse* }\end{array}$ & $\begin{array}{l}\text { Yes } \\
\text { No }\end{array}$ & $\begin{array}{l}\text { Within } 48 \text { hours of onset } \\
\text { of symptoms }\end{array}$ & $\begin{array}{l}\text { Electronic patient } \\
\text { record }\end{array}$ \\
\hline
\end{tabular}

*Indicator that will used to determine the maintenance of the intervention.

ADL, activities of daily living; CAR, cardiology; GER, geriatrics; n/a, not available.

focus groups will be interviewed individually. The experiences of participating patients will be captured using structured patient interviews. The sampled experiences of healthcare professionals and patients will be used to determine the acceptability and to assess for barriers and facilitators of both the intervention and implementation strategy.

\section{Effectiveness evaluation}

Baseline variables

The baseline evaluation of control and intervention patients serves to assess baseline equivalence between patients in the before and after cohorts for the outcome evaluation (table 4). Demographic data will be collected on age, gender, living situation and use of healthcare services using patient interview or review of the electronic record. Medical variables include the medical diagnoses, number and type of medications and comorbidities. ${ }^{27}$ The following variables related to functional status will be measured: (in)dependence on activities of daily living (ADL),${ }^{28}{ }^{29}$ instrumental ADL,${ }^{30}$ community mobility, ${ }^{31}$ physical performance, ${ }^{32}$ handgrip strength, ${ }^{33}$ fall history ${ }^{34}$ and physical frailty. ${ }^{35}$ Regarding mental status, presence of cognitive impairment, ${ }^{36}$ depression, ${ }^{37}$ anxiety ${ }^{38}$ and delirium ${ }^{39}$ will be measured. Finally, nutritional status will be assessed using the MNA-Short Form. ${ }^{40}$

\section{Outcome variables}

Functional decline is the primary outcome of interest measured by comparing the Katz ADL score on hospital admission, hospital discharge and at 1, 3 and 6 months follow-up. ${ }^{24}{ }^{25}$ An increase of 1 point on the Katz Index will be considered clinically relevant to define functional decline. Secondary outcomes are community mobility assessed at 1, 3 and 6 months follow-up measured with Life-Space Assessment and physical performance at hospital discharge measured with the Short Physical Performance Battery. ${ }^{31} 32$

Incident in-hospital geriatric syndromes include delirium, cognitive decline, falls and obstipation. Delirium will be operationalised using the three-dimensional-Confusion Assessment Method (CAM) after a trained researcher assessed cognitive functioning using the CAM questionnaire on day 1 (day of admission), 3, 5, 7 and 9 (or daily

Table 3 Dose indicators

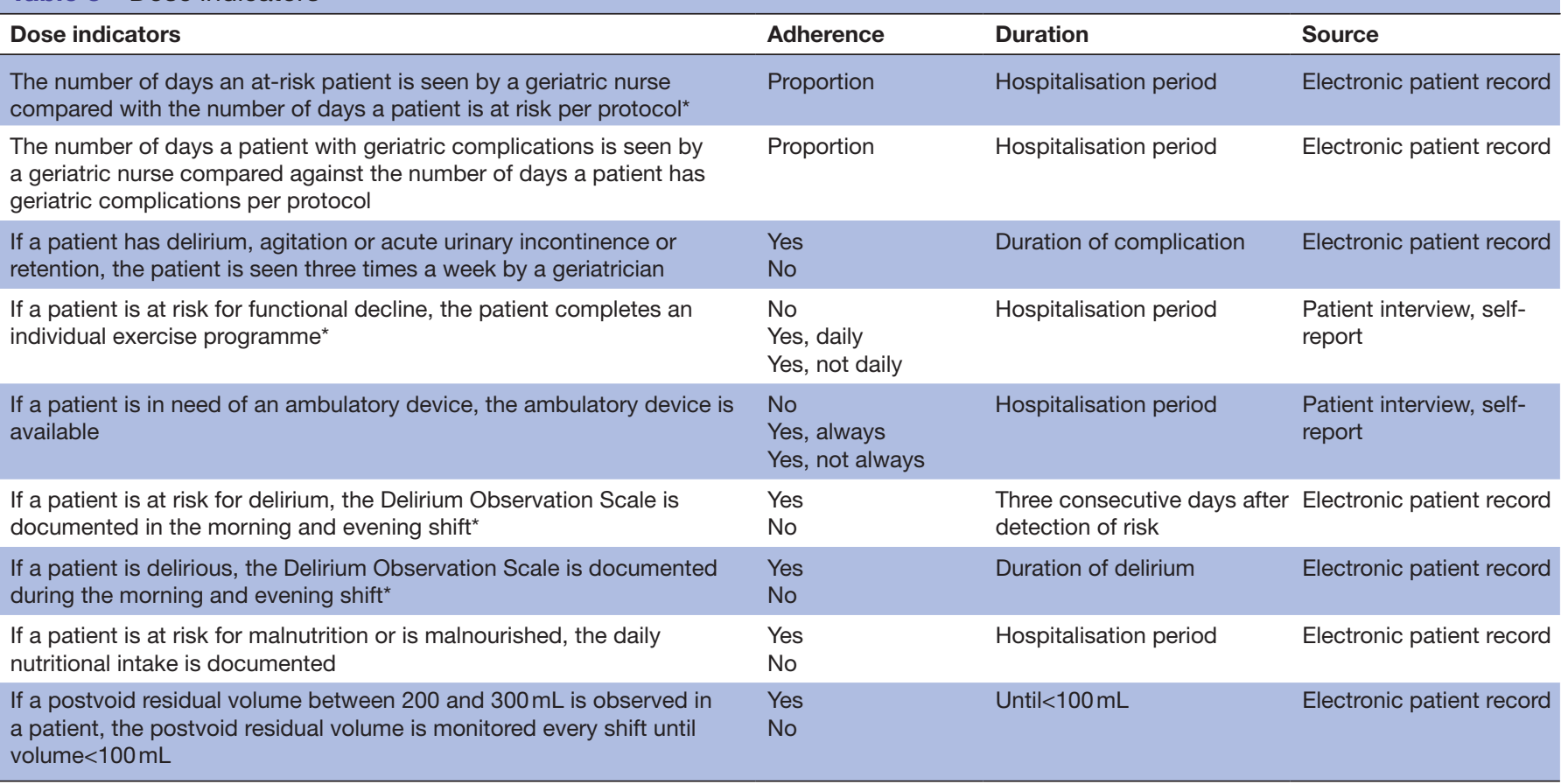

*Indicator that will used to determine the maintenance of the intervention. 


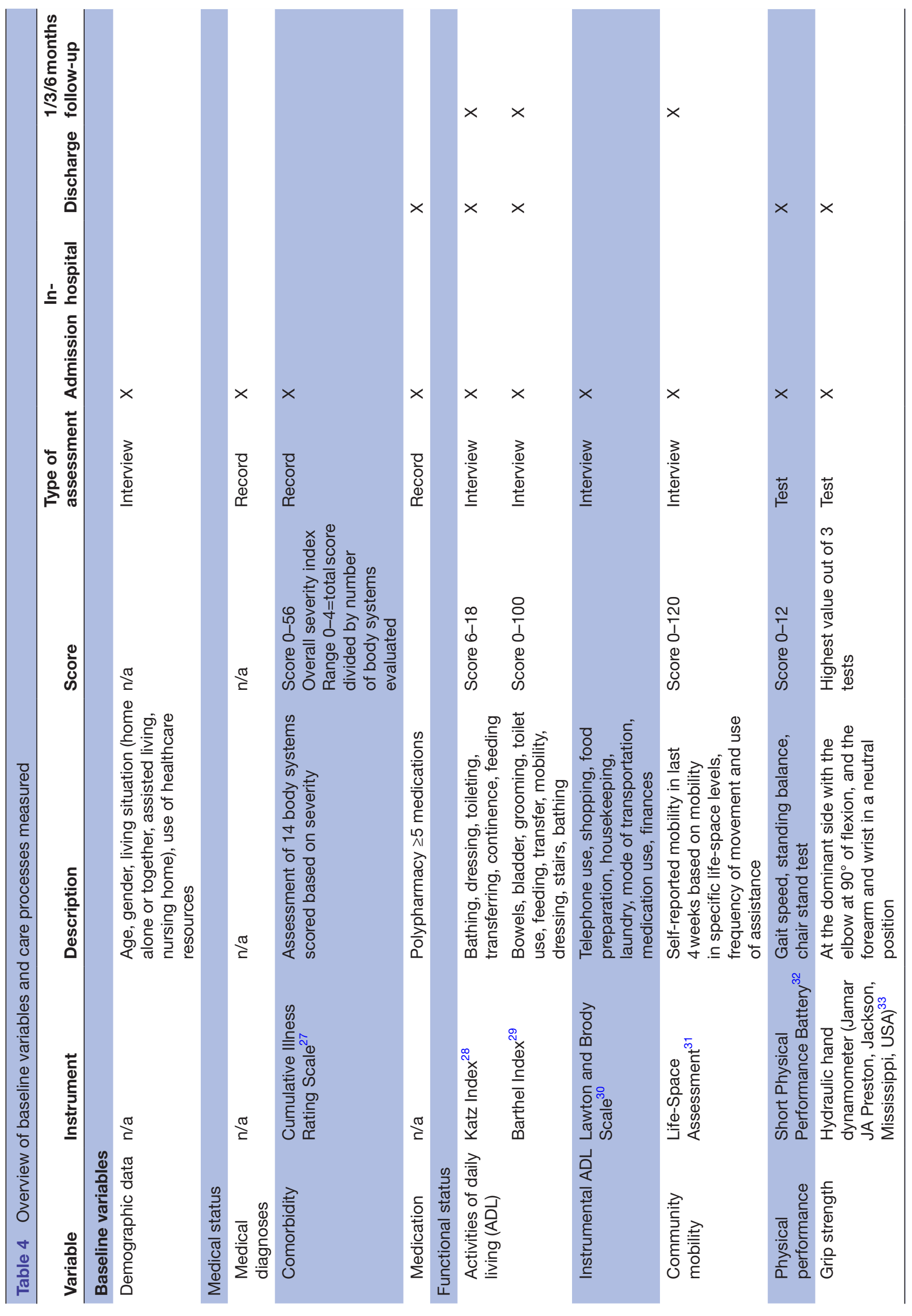




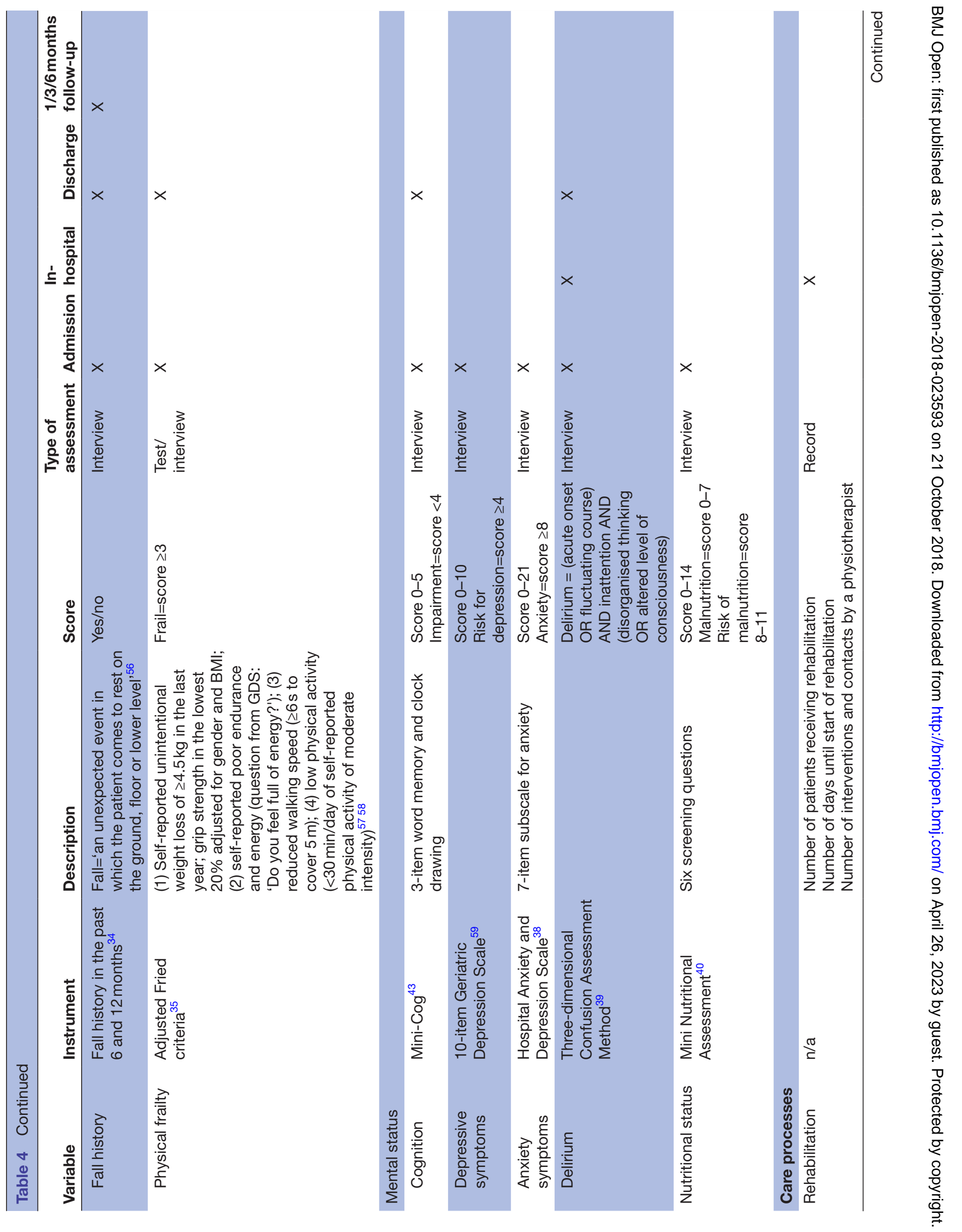




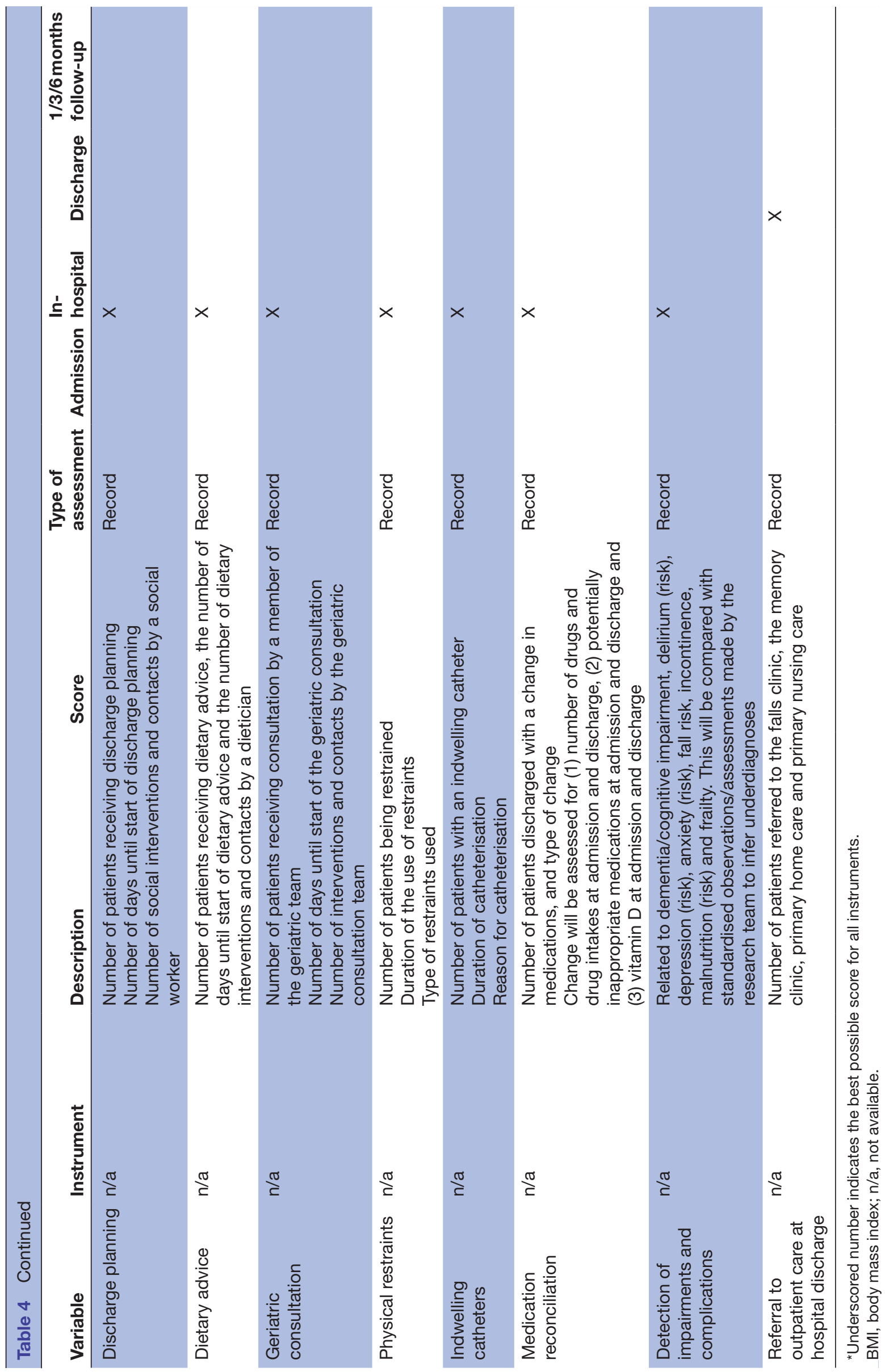


in delirious patients). ${ }^{41} 42$ Patients are considered delirious based on the sensitive CAM algorithm criteria. The duration of delirium will be determined as the number of days from the first positive CAM score until the day before a negative CAM score was obtained ${ }^{36}$ In-hospital cognitive decline will be determined by a decline on the Mini-Cog score between hospital admission and discharge. ${ }^{43}$ Symptomatic infections will be assessed by reviewing the patient record for antibiotic treatment for a clinical infection (eg, lower respiratory tract infection, urinary tract infection, skin and soft tissue infection, infection of unknown origin and sepsis without primary focus).

Obstipation defined as 'not having passed stool in 5 days or more' will be assessed by reviewing the patient record for nurses recorded observations (which are assessed every shift). In-hospital falls and fall-related injuries will be monitored using the patient record, while postdischarge falls and fall-related injuries will be monitored at 1, 3 and 6 months follow-up by telephone.

Length of hospital stay will be measured in days and hours for admission on the cardiology unit and non-cardiology unit. Unplanned readmission rate will be assessed at 1, 3 and 6 months follow-up by telephone and by checking the electronic patient file. To be considered unplanned, patients should be admitted through the emergency department or outpatient clinic. Mortality will be assessed in-hospital using the electronic patient record, and at 1, 3 and 6 months follow-up by telephone. Institutionalisation, defined as a new admission to a long-term care facility compared with baseline, will be assessed at discharge and on 1, 3 and 6 months follow-up by telephone. Quality of life will be assessed using the 5-level EQ-5D on hospital admission, hospital discharge and at 1, 3 and 6 months follow-up. ${ }^{445}$

\section{Process evaluation}

A process evaluation will be embedded in the after cohort of the evaluation study to determine how the process of care was changed as a result of the implementation of the intervention and how the intervention was maintained and adapted over time and how this related to the interaction between context factors and the implementation of the intervention. The change in process of care will be observed using the electronic patient record and include the use, time to start and frequency of geriatric support services, physical therapy, discharge planning and nutritional advice, the use and duration of physical restraints and indwelling catheters, the detection of geriatric syndromes, medication reconciliation and referral to outpatient services. The maintenance of the intervention relates to how well the reach, fidelity and dose of the intervention is maintained over time, which will be monitored using the electronic patient record (see selection of indicators in tables 2 and 3). Adaptations to the intervention will be monitored by the study coordinator during the monthly working group meetings with stakeholders. Focus groups and interviews will be organised to sample the experiences of all healthcare professionals participating in the intervention. The experiences will focus on how contextual factors influenced the maintenance and adaptations of the intervention and how this relates to the sustainability of the intervention.

\section{Sample size}

Feasibility evaluation

A total of 30 consecutive patients receiving the intervention will be recruited for the feasibility study. Approximately 30 healthcare professionals will be recruited for the focus groups and interviews. The total sample will be based on the willingness to participate and data saturation.

\section{Effectiveness evaluation}

A sample size has been calculated for in-hospital functional decline, the primary outcome of the evaluation study. We assumed a minimal important difference of 1 mean point on the Katz ADL and a SD of 3 points on the Katz ADL with equal groups, based on observations in a pilot study. ${ }^{22}$ This equals a standardised effect size of 0.33 (Cohen's d) and indicates a low-to-moderate effect size. Therefore, a total of 159 patients are needed per group ( $\alpha=0.05$, Power $=0.8$, two-sided test), accounting for $10 \%$ missing data. However, we hypothesised that not all patients will benefit from the intervention as several studies have identified larger effects sizes in patients with premorbid impairments but sufficient capacity to participate in in-hospital interventions. ${ }^{46-50}$ Based on these studies, we expect that $30 \%$ of the patients will be at low risk, $50 \%$ at high risk and that $20 \%$ will have an acute problem. This means that 227 patients need to be assessed to be able to evaluate the geriatric co-management intervention in 159 patients in the high-risk $(\mathrm{n}=114)$ or acute problem group $(\mathrm{n}=45)$.

\section{Process evaluation}

The process evaluation is embedded in the sample of patients recruited for the effectiveness evaluation. A comprehensive sample of all healthcare professionals with at least 4 weeks of exposure to the intervention will be recruited, with the total sample depending on the willingness to participate and data saturation.

\section{Data collection procedure}

Feasibility evaluation

Researchers will recruit patients on hospital admission after written (proxy-)informed consent has been obtained and will monitor the feasibility indicators using the electronic patient record daily and by bedside assessment every other day. Patients are interviewed on hospital discharge by a researcher using a structured patient questionnaire. At the end of the feasibility phase, focus group discussions will be organised. One researcher will coordinate the group discussions and a second researcher will take notes. Healthcare professionals not able to participate in group discussions will be interviewed individually. An interview guide will be composed based on a literature search for existing barriers and facilitators and the role of 
contextual factors. All discussions will be tape recorded and written out verbatim. The audio recordings will be deleted and only the verbatim text will be saved.

\section{Effectiveness evaluation}

In the before and after cohorts, patients are recruited on hospital admission by the researchers, who screen the patient records for eligibility criteria and obtain written (proxy-)informed consent in a face-to-face interview. A research assistant will monitor the incidence of complications using patient assessment and by monitoring the patient record throughout hospitalisation, and will assess the outcomes on hospital discharge using patient interview. Patients will receive a letter by post with instructions and an assessment questionnaire for follow-up assessment at 1, 3 and 6 months postdischarge. Researchers will contact the patient by telephone to complete the assessment. Due to the nature of the intervention and study design, health professionals and patients cannot be blinded. Blinding of outcome assessors is not considered feasible due to limited resources.

\section{Process evaluation}

The data collection procedure for the process evaluation is equal to one of the feasibility evaluation, but only a selection of fidelity and dose indicators will be measured for all patients in the after cohort.

\section{Data management and monitoring}

Standardised data collection forms will be drafted and piloted by all researchers. Databases will be drafted in Excel and SPSS and all researchers will have access to a codebook. The study coordinator will assess the integrity of all completed informed consents and will monitor the assessment documents for missing data. Written assessments will be recorded in an Excel and SPSS database on a password-protected computer, and will be analysed for wild codes and extreme values. All data will be coded and analysed anonymously. A formal data monitoring committee is not considered necessary as the study duration is relatively short and the risks for patients are considered minimal. Interim analyses and stopping rules have not been defined. Researchers will be trained to monitor for and record adverse events during assessments and tests, which will always be performed in proximity of a licensed health professional.

\section{Statistical methods, qualitative analysis and data integration}

Variables will be explored using visual and descriptive statistics and analysed for missing data. Categorical data will be expressed as number of cases and percentages. Continuous data will be expressed as means with SD. All primary analyses will be conducted on the patients who were at high risk for functional decline or patients experiencing an acute problem. For evaluating the primary outcome, we will first explore the baseline equivalence between the control and intervention group. If equivalent, we will test the absolute difference in ADL scores on hospital discharge between the two groups. If not equivalent, we will test the mean decline in ADL between hospital admission and discharge in both group. The analysis of covariance (ANCOVA) model will be used to adjust for confounders. For secondary outcomes, logistic regression will be used for dichotomous outcomes, survival analyses for time to event variables and ANCOVA for mean differences between groups. We will explore several moderating variables. We hypothesise that the effect of the intervention will be dependent on (1) the baseline risk of patients for developing functional decline, (2) the fidelity and dose of the implementation and intervention and (3) the presence of heart failure. Results will not be corrected for multiple testing. Statistical inference will be based on 95\% CIs.

Focus group discussions and individual interviews will be analysed using a thematic analysis to understand how experiences influenced the implementation and feasibility of the intervention. Two researchers will independently code the data using Word documents. Transcripts and results will not be returned to participants for feedback. The following strategies will be used to support the methodological quality: peer review, triangulation, audit trial, methodological and reflective notes and thick description.

Integration of quantitative and qualitative data will be done through embedding. ${ }^{51}$ Data collection and analysis will be recurrently linked at multiple points: in the development phase to clarify outcome measures, in the evaluation phase to understand contextual factors that influence the study findings and in the postevaluation phase to explain outliers or develop hypotheses about necessary changes for large-scale implementation. Quantitative and qualitative data will be integrated in a narrative way using a contiguous approach, meaning that findings will be presented in a single report in different sections. In case qualitative and quantitative findings are inconsistent, contradict or conflict, we will reanalyse the existing databases to resolve differences, seek explanations from theory or further analyse discordance in follow-up studies. ${ }^{51}$

\section{Ethics and dissemination}

Written voluntary (proxy-)informed consent will be obtained from all participants at the start of the study. On each assessment, the research assistant will obtain oral informed consent for the assessment. Patients will be considered the owners of their data, and data will be removed or changed on the request of the patient. No financial compensation is rewarded for participation, and patients are not charged any costs as a result of any action in this study. Dissemination of the results will be through articles in scientific and professional journals both in English and Dutch and by conference presentations. A G-COACH publication policy has been developed and was approved on the first consortium meeting.

\section{DISCUSSION}

This paper presents the study design and methods of the G-COACH intervention study, which is to our knowledge the first study evaluating the feasibility and effectiveness 
of a geriatric co-management intervention in older cardiology patients. In view of the rapidly increasing number of hospitalised older patients and the continuous efforts to further improve quality of care for these frail and complex patients, this study is timely and needed.

We hypothesise that our framework of geriatric co-management will be beneficial in this population, because of the applied methodological framework. First, a theoretical geriatric co-management model was developed by integrating evidence from a meta-analysis, quality indicators and a prospective cohort study. ${ }^{182122}$ Such a theoretical model details how the intervention will impact the desired outcomes, and increases the a priori probability for a clinically meaningful effect. ${ }^{52}$ Second, important stakeholders will be involved in translating the theoretical care model in an operational geriatric co-management programme. ${ }^{53}$ Therefore, physicians, nurses, allied healthcare workers and nursing, medical and administrative management are involved in the development, feasibility and evaluation phase of the project. This will allow us to implement and evaluate a care programme that fits the local context of the hospital and the participating units, hence, a programme that is deemed beneficial, acceptable and feasible by all stakeholders involved. Third, we will formally test the feasibility of a geriatric co-management programme. By first testing the feasibility, the intervention can be adjusted and optimised before investing in a large-scale evaluation. ${ }^{20}{ }^{54}$ This approach contrasts with the majority of studies in which feasibility problems are detected in evaluation studies leading to inconclusive results. Finally, because information is currently missing on what components make geriatric co-management effective in order to replicate the observed effects in daily practice, we will evaluate geriatric co-management using a mixed-methods design. By incorporating quantitative and qualitative information in both the outcome and process evaluation, we can move beyond effect outcomes and understand how intervention components interact with context and system factors to derive an effect on patient outcomes. ${ }^{51}$ This will help us understand why geriatric co-management worked or-in case the intervention would not be successful-why it did not work. The study will therefore in any case add to the evidence-base regarding the development, evaluation and implementation of geriatric co-management programmes.

Despite the absence of strong evidence regarding the impact of geriatric co-management in a recent meta-analysis, ${ }^{18}$ we have deliberately chosen to use a hybrid 1 effectiveness-implementation design. This is one of the three hybrid designs described by Curran et al who mapped different implementation research designs. ${ }^{23}$ By systematically addressing the healthcare needs, preferences and values at different levels (ie, patient, provider, system and policy level) and by engaging relevant stakeholders, implementation research effectively brings evidencebased models into practice in a context-sensitive way leading to sustainable change. While large-scale implementation is outside the scope of the G-COACH project, we will actively explore components that will facilitate future implementation of the care model if it proves to be successful by: (1) defining core intervention components that are essential for all co-management programmes and defining peripheral components that can be adapted to the local context; (2) describing how context factors influenced the processes of geriatric co-management; (3) describing how participants experienced geriatric co-management and how this influenced adopting the programme locally; (4) evaluating how well geriatric co-management was implemented on the participating units. ${ }^{55}$ Addressing these knowledge gaps is essential before considering scaling up and scaling out the geriatric co-management model of care.

In conclusion, the G-COACH intervention study will be the first to evaluate the impact of cardiogeriatric co-management and has the potential to change the current clinical practice of frail older hospitalised patients.

\section{Trial status}

Data for the 227 patients in the before cohort was collected between 20 September 2016 and 27 June 2017. The feasibility study was conducted between 28 June and 31 December 2017. Data for the 227 patients in the after cohort commenced on 1 January 2018 and is expected to continue until October 2018.

\section{Author affiliations}

${ }^{1}$ Gerontology and Geriatrics, Department of Chronic Diseases, Metabolism and Ageing, KU Leuven — University of Leuven, Leuven, Belgium

${ }^{2}$ Nursing Science, Department of Public Health, University of Basel, Basel,

Switzerland

${ }^{3}$ Research Foundation, Flanders, Belgium

${ }^{4}$ Department of Public Health and Primary Care, University of Leuven - KU Leuven, Leuven, Belgium

${ }^{5}$ Department of Geriatric Medicine, University Hospitals Leuven, Leuven, Belgium ${ }^{6}$ Department of Cardiovascular Diseases, KU Leuven—University of Leuven, University Hospitals Leuven, Leuven, Belgium

${ }^{7}$ Department of Anesthesiology, University Hospitals Leuven, Leuven, Belgium

Contributors All authors made significant contribution to the conception and design of the study protocol. MD and BVG designed the original concept and wrote the study protocol and manuscript. The protocol and manuscript was critically reviewed by AJ, ED, BDdC, CD, KF, M-CH, MH, BM, SR, JT, KM and JF. BVG wrote the statistical analysis plan. MD is the principal investigator and BVG is the study coordinator of the G-COACH project. All authors gave approval for the publication.

Funding This study was funded by the KU Leuven Research Council (REF 22/15/028; G-COACH) and the Viviane Conraads Award 2015.

Disclaimer The KU Leuven Research Council had no role in the design of the study and collection, analysis and interpretation of data and in writing the manuscript.

Competing interests None declared.

Patient consent Not required.

Ethics approval The study protocol was approved by the Medical Ethics Committee of UZ/KU Leuven (S58296).

Provenance and peer review Not commissioned; externally peer reviewed.

Open access This is an open access article distributed in accordance with the Creative Commons Attribution Non Commercial (CC BY-NC 4.0) license, which permits others to distribute, remix, adapt, build upon this work non-commercially, and license their derivative works on different terms, provided the original work is properly cited, appropriate credit is given, any changes made indicated, and the use is non-commercial. See: http://creativecommons.org/licenses/by-nc/4.0/. 


\section{REFERENCES}

1. Rechel B, Grundy E, Robine JM, et al. Ageing in the European Union. Lancet 2013;381:1312-22.

2. Sánchez E, Vidán MT, Serra JA, et al. Prevalence of geriatric syndromes and impact on clinical and functional outcomes in older patients with acute cardiac diseases. Heart 2011;97:1602-6.

3. Huerre C, Guiot A, Maréchaux S, et al. Functional decline in elderly patients presenting with acute coronary syndromes: impact on midterm outcome. Arch Cardiovasc Dis 2010;103:19-25.

4. Chaudhry SI, Wang Y, Gill TM, et al. Geriatric conditions and subsequent mortality in older patients with heart failure. J Am Coll Cardiol 2010;55:309-16.

5. Milisen K, Dejaeger E. Geriatric and gerontologic nursing: investing in quantity or quality?. Tijdschr Gerontol Geriatr 2006;37:86-8.

6. Vasilevskis EE, Simmons SF. Simple solutions may not work for complex patients: a need for new paradigms in geriatric hospital medicine. J Hosp Med 2015;10:343-4.

7. Rohrer JE, Garrison G, Oberhelman SA, et al. Epidemiology of polypharmacy among family medicine patients at hospital discharge. J Prim Care Community Health 2013;4:101-5.

8. Inouye SK, Westendorp RG, Saczynski JS. Delirium in elderly people. Lancet 2014;383:911-22.

9. Boustani M, Baker MS, Campbell N, et al. Impact and recognition of cognitive impairment among hospitalized elders. J Hosp Med 2010;5:69-75.

10. Cepoiu M, McCusker J, Cole MG, et al. Recognition of depression in older medical inpatients. J Gen Intern Med 2007;22:559-64.

11. Wilkins E, Wilson L, Wickramasinghe K, et al. European Cardiovascular Disease Statistics 2017. Brussels, Belgium, 2017.

12. Forman DE, Rich MW, Alexander KP, et al. Cardiac care for older adults. Time for a new paradigm. J Am Coll Cardiol 2011;57:1801-10.

13. Azad NA, Mielniczuk L. A call for collaboration: improving cardiogeriatric care. Can J Cardiol 2016;32:1041-4.

14. Ellis G, Gardner M, Tsiachristas A, et al. Comprehensive geriatric assessment for older adults admitted to hospital. Cochrane Database Syst Rev 2017;9:CD006211.

15. Ellis G, Langhorne P. Comprehensive geriatric assessment for older hospital patients. Br Med Bull 2004;71:45-59.

16. Deschodt M, Flamaing J, Haentjens P, et al. Impact of geriatric consultation teams on clinical outcome in acute hospitals: a systematic review and meta-analysis. BMC Med 2013;11:48.

17. Deschodt M, Claes V, Van Grootven B, et al. Comprehensive Geriatric Care in Hospitals: the role of inpatient geriatric consultation teams: KCE rapport. 2015.

18. Van Grootven B, Flamaing J, Dierckx de Casterlé B, et al. Effectiveness of in-hospital geriatric co-management: a systematic review and meta-analysis. Age Ageing 2017;46:903-10.

19. Chan AW, Tetzlaff JM, Gøtzsche PC, et al. SPIRIT 2013 explanation and elaboration: guidance for protocols of clinical trials. BMJ 2013;346:e7586.

20. Craig P, Dieppe P, Macintyre S, et al. Developing and evaluating complex interventions: the new Medical Research Council guidance. BMJ 2008;337:a1655.

21. Van Grootven B, McNicoll L, Mendelson DA, et al. Quality indicators for in-hospital geriatric co-management programmes: a systematic literature review and international Delphi study. BMJ Open 2018;8:e020617

22. Jonckers M, Van Grootven B, Willemyns E, et al. Hospitalizationassociated disability in older adults with valvular heart disease: incidence, risk factors and its association with care processes. Acta Cardiol 2018:1-7.

23. Curran GM, Bauer M, Mittman B, et al. Effectiveness-implementation hybrid designs: combining elements of clinical effectiveness and implementation research to enhance public health impact. Med Care 2012;50:217-26

24. Moore GF, Audrey S, Barker M, et al. Process evaluation of complex interventions: Medical Research Council guidance. BMJ 2015;350:h1258.

25. Deschodt M, Braes T, Broos $\mathrm{P}$, et al. Effect of an inpatient geriatric consultation team on functional outcome, mortality, institutionalization, and readmission rate in older adults with hip fracture: a controlled trial. J Am Geriatr Soc 2011;59:1299-308.

26. Kok G, Gottlieb NH, Peters GJ, et al. A taxonomy of behaviour change methods: an intervention mapping approach. Health Psychol Rev 2016;10:297-312.

27. Salvi F, Miller MD, Grilli A, et al. A manual of guidelines to score the modified cumulative illness rating scale and its validation in acute hospitalized elderly patients. J Am Geriatr Soc 2008;56:1926-31.

28. Katz S, Akpom CA. 12. Index of ADL. Med Care 1976;14:116-8.
29. Mahoney FI, Barthel DW. Functional evaluation: the barthel index. Md State Med J 1965;14:61-5.

30. Lawton MP, Brody EM. Assessment of older people: selfmaintaining and instrumental activities of daily living. Gerontologist 1969;9:179-86.

31. Baker PS, Bodner EV, Allman RM. Measuring life-space mobility in community-dwelling older adults. J Am Geriatr Soc 2003;51:1610-4.

32. Guralnik JM, Simonsick EM, Ferrucci L, et al. A short physica performance battery assessing lower extremity function: association with self-reported disability and prediction of mortality and nursing home admission. J Gerontol 1994:49:M85-94.

33. Pitta F, Troosters T, Probst VS, et al. Are patients with COPD more active after pulmonary rehabilitation? Chest 2008;134:273-80.

34. Milisen K, Coussement J, Flamaing J, et al. Fall prediction according to nurses' clinical judgment: differences between medical, surgical, and geriatric wards. J Am Geriatr Soc 2012;60:1115-21.

35. Fried LP, Tangen CM, Walston J, et al. Frailty in older adults: evidence for a phenotype. J Gerontol A Biol Sci Med Sci 2001;56:M146-56.

36. Borson S, Scanlan JM, Chen P, et al. The Mini-Cog as a screen for dementia: validation in a population-based sample. J Am Geriatr Soc 2003;51:1451-4.

37. Jongenelis K, Pot AM, Eisses AM, et al. Diagnostic accuracy of the original 30-item and shortened versions of the geriatric depression scale in nursing home patients. Int J Geriatr Psychiatry 2005;20:1067-74.

38. Zigmond AS, Snaith RP. The hospital anxiety and depression scale. Acta Psychiatr Scand 1983;67:361-70.

39. Marcantonio ER, Ngo LH, O'Connor M, et al. 3D-CAM: derivation and validation of a 3-minute diagnostic interview for CAM-defined delirium: a cross-sectional diagnostic test study. Ann Intern Med 2014;161:554-61.

40. Kaiser MJ, Bauer JM, Ramsch C, et al. Validation of the Mini Nutritional Assessment short-form (MNA-SF): a practical tool for identification of nutritional status. J Nutr Health Aging 2009;13:782-8.

41. Inouye SK, van Dyck $\mathrm{CH}$, Alessi CA, et al. Clarifying confusion: the confusion assessment method. A new method for detection of delirium. Ann Intern Med 1990;113:941-8.

42. Palihnick K, Inouye SK, Marcantonio ER. The 3D CAM Training Manual for Research. Boston: Hospital Elder Life Programme, 2014.

43. McCarten JR, Anderson P, Kuskowski MA, et al. Screening for cognitive impairment in an elderly veteran population: acceptability and results using different versions of the Mini-Cog. J Am Geriatr Soc 2011:59:309-13.

44. van Reenen M, Janssen B, 2015. EQ-5D-5L User Guide. Basic information on how to use the EQ-5D-5L instrument. http://www. euroqol.org/fileadmin/user_upload/Documenten/PDF/Folders_Flyers/ EQ-5D-5L_UserGuide_2015.pdf (accessed 24 Jul 2016).

45. Herdman M, Gudex C, Lloyd A, et al. Development and preliminary testing of the new five-level version of EQ-5D (EQ-5D-5L). Qual Life Res 2011;20:1727-36.

46. Prestmo A, Saltvedt I, Helbostad JL, et al. Who benefits from orthogeriatric treatment? Results from the Trondheim hip-fracture trial. BMC Geriatr 2016;16:49.

47. Kennie DC, Reid J, Richardson IR, et al. Effectiveness of geriatric rehabilitative care after fractures of the proximal femur in elderly women: a randomised clinical trial. BMJ 1988;297:1083-6.

48. Huusko TM, Karppi P, Avikainen V, et al. Randomised, clinically controlled trial of intensive geriatric rehabilitation in patients with hip fracture: subgroup analysis of patients with dementia. BMJ 2000;321:1107-11.

49. Bogardus ST, Desai MM, Williams CS, et al. The effects of a targeted multicomponent delirium intervention on postdischarge outcomes fo hospitalized older adults. Am J Med 2003;114:383-90.

50. Tinetti ME, Baker DI, McAvay G, et al. A multifactorial intervention to reduce the risk of falling among elderly people living in the community. N Engl J Med 1994;331:821-7.

51. Fetters MD, Curry LA, Creswell JW. Achieving integration in mixed methods designs-principles and practices. Health Serv Res 2013;48:2134-56.

52. Bartholomew LK, Parcel GS, Kok G. Intervention mapping: a process for developing theory- and evidence-based health education programs. Health Educ Behav 1998;25:545-63.

53. Rycroft-Malone J, Wilkinson J, Burton CR, et al. Collaborative action around implementation in Collaborations for Leadership in Applied Health Research and Care: towards a programme theory. J Health Serv Res Policy 2013;18:13-26. 
54. Lancaster GA, Dodd S, Williamson PR. Design and analysis of pilot studies: recommendations for good practice. J Eval Clin Pract 2004;10:307-12.

55. Damschroder LJ, Aron DC, Keith RE, et al. Fostering implementation of health services research findings into practice: a consolidated framework for advancing implementation science. Implement Sci 2009;4:50.

56. Lamb SE, Jørstad-Stein EC, Hauer K, et al. Development of a common outcome data set for fall injury prevention trials: the prevention of falls network Europe consensus. J Am Geriatr Soc 2005;53:1618-22.
57. Afilalo J. Frailty in patients with cardiovascular disease: why, when, and how to measure. Curr Cardiovasc Risk Rep 2011;5:467-72.

58. Mossello E, Profili F, Di Bari M, et al. Postal screening can identify frailty and predict poor outcomes in older adults: longitudinal data from INTER-FRAIL study. Age Ageing 2016;45:469-74.

59. Almeida OP, Almeida SA. Short versions of the geriatric depression scale: a study of their validity for the diagnosis of a major depressive episode according to ICD-10 and DSM-IV. Int J Geriatr Psychiatry 1999;14:858-65. 